THE EXTENDED IONIZED NEBULOSITIES SURROUNDING THE ACTIVE GALAXIES NGC 6215, A 0945-30 AND MCG-2-58-22

\author{
Florence DURRET \\ Institut d'Astrophysique de Paris \\ $98^{\text {bis }} \mathrm{B}^{d}$ Arago \\ F - 75014 PARIS, FRANCE
}

\title{
OBSERVATIONAL RESULTS
}

The following results are fully described in Durret and Bergeron (1988).

NGC 6215 is a radio-loud spiral galaxy at a distance of $31.3 \mathrm{Mpc}$ (with $\mathrm{H}_{o}=$ $50 \mathrm{~km} \mathrm{~s} \mathrm{~s}^{-1} \mathrm{Mpc}^{-1}$ throughout), located $165 \mathrm{kpc}$ away from the Seyfert 2 NGC 6221 and embedded in an ionized nebulosity of diameter $8.3 \mathrm{kpc}$ along $\mathrm{PA}=340^{\circ}$. This gaseous envelope is three times more extended towards the South than towards the North. The nucleus of NGC 6215 has characteristics of a weak LINER (Low Ionization Nuclear Emitting Region, see Heckman, 1980), with [OI] $\lambda 6300$ detected at a $3 \sigma$ level. The extranuclear spectra are indistinguishable from HII region type, and all the zones, including the nucleus, have very similar excitation levels. The ionized gas shows no velocity gradient along $\mathrm{PA}=340^{\circ}: \Delta v<50 \mathrm{~km} \mathrm{~s}^{-1}$, suggesting that the minor axis of the nebulosity is close to the photometric minor axis.

A 0945-30(=MCG-5-23-16) is an So/a galaxy, located at a distance of $50.8 \mathrm{Mpc}$; it is variable in $\mathrm{X}$-rays but is not a radio source. The ionized envelope around this galaxy has a diameter of $8.5 \mathrm{kpc}$ along $\mathrm{PA}=232^{\circ}$, and is symmetrically distributed on either side of the nucleus. Its nuclear spectrum is that of a high excitation narrow line Seyfert, with $[\mathrm{OIII}] \lambda 5007 / \mathrm{H} \beta \simeq 17,[\mathrm{NeV}] \lambda 3426 /[\mathrm{NeIII}] \lambda 3869 \simeq 1$ and HeII $\lambda 4686$ present, together with $[\mathrm{OI}] \lambda 6300$. A synthesis analysis of the $\mathrm{H} \alpha-$ [NII] blend shows the existence of a weak broad component in $\mathrm{H} \alpha$, implying that this galaxy is in fact a Seyfert 1.9. The extranuclear regions are of lower excitation than the nucleus, particularly to the south-west. This extended nebulosity is one of the few examples where [OII] $\lambda 3727$ is detected further away from the nucleus than $[\mathrm{OIII}] \lambda 5007$. The velocity field of the ionized gas is consistent with solid body rotation throughout, with a maximum amplitude of $450 \mathrm{~km} \mathrm{~s} \mathrm{~s}^{-1}$ and a velocity gradient of $68 \mathrm{~km} \mathrm{~s}^{-1} \mathrm{kpc}^{-1}$.

MCG-2-58-22 (= Mkn 926) is a Seyfert 1 located at a distance of $277 M p c$, with extremely broad Balmer lines, a very high $\mathrm{X}$-ray luminosity and a barely resolved central radio source. It is surrounded by a very large ionized nebulosity of diameter $47 \mathrm{kpc}$ along $\mathrm{PA}=270^{\circ}$, one of the largest detected around Seyfert 1s. As in A 0945-30, the gas is of rather unusually low excitation outside the nucleus, with I $([\mathrm{OII}] \lambda 3727)$ notably larger than $\mathrm{I}([\mathrm{OIII}] \lambda 5007)$. The velocity field of the ionized gas has an extremely large amplitude of about $800 \mathrm{~km} \mathrm{~s}^{-1}$. Solid body rotation is observed over the central $28 \mathrm{kpc}$, with a gradient of $24 \mathrm{~km} \mathrm{~s} \mathrm{~s}^{-1} \mathrm{kpc}^{-1}$, and a flattening of the rotation curve in the easternmost region. 


\section{DISCUSSION}

By what kind of radiation is the gas ionized in the nuclear and extranuclear regions?

The emission line intensities of the nuclear and extranuclear regions of NGC 6215 cannot be simultaneously fitted by HII region models (e.g. Stasinska, 1980). On the other hand, they are consistent with those predicted for LINERs by Binette (1985), where the gas of density $400 \mathrm{~cm}^{-3}$ is photoionized by a power-law continuum $F_{\nu} \propto \nu^{-2.0}$, and has an average ionization parameter $U \simeq 10^{-4}$ (within a factor of 2). The gas in NGC 6215 therefore does seem to be ionized by a weak active nucleus, whose UV radiation is responsible for the ionization of the nuclear and possibly also extranuclear regions.

The nuclear spectrum of A $\mathbf{0 9 4 5 - 3 0}$ is that of a high excitation Seyfert 1.9, which we believe to be an obscured Seyfert 1. A comparison of its nuclear emission line intensities with photoionization models by Stasinska (1984) gives a satisfactory fit for a blackbody ionizing radiation of effective temperature $210^{5} \mathrm{~K}$, an ionization parameter $\mathrm{U}=(1-2) 10^{-3}$, a gas density $\mathrm{n}=(1-2) 10^{4} \mathrm{~cm}^{-3}$ and abundances close to solar. The gas north-east of the nucleus is probably photoionized by a similar UV radiation of somewhat smaller effective temperature $1.510^{5} \mathrm{~K}$, an has a lower value of $U=(2-4) 10^{-4}$. The gas south-west of the nucleus seems of lower excitation and redder, implying the presence of dust which could prevent the nuclear ionizing continuum from reaching this zone; the gas could in that region be photoionized either by partially obscured UV radiation coming from the nucleus, or by nearby hot stars.

The nuclear spectrum of the Seyfert 1 galaxy MCG-2-58-22 is of notably lower excitation than that of A $0945-30$. Since only [OlI] $\lambda 3727$ and sometimes $\mathrm{H} \beta$ are detected outside the nucleus, we cannot discuss the ionization mechanism and physical properties of the extranuclear gas.

Are there correlations between the ionized nebulosity extent and other properties?

Very large ionized nebulosities (of diameter larger than 20-30 kpc) seem to have been until now preferably found (see e.g. Fosbury, 1986) around :

- Seyfert galaxies or quasars rather than LINERs

- radio loud objects

- intrinsically bright active galactic nuclei, particularly in the UV

The analysis of a catalogue of 157 ionized nebulosities found in the litterature (Durret, unpublished) confirms that :

- few gaseous envelopes are found around LINERs (10 out of 157 , or $6 \%$ )

- 115 out of $157(75 \%)$ nebulosities are indeed observed around radio-loud AGN, but no clear correlation is found between the diameter of the ionized gas and the radio flux

- ionized envelopes do tend to be larger around AGN with bright absolute magnitudes, but this is a trend rather than a correlation.

\section{REFERENCES}

Binette, L. : 1985, Astronomy and Astrophysics, 143, 334

Durret, F., Bergeron, J. : 1988, Astronomy and Astrophysics, in press and IAP Preprint $n^{\circ} 235$

Fosbury, R.A.E. : 1986, in Proc. Trieste Meeting "Structure and Evolution of Active Galactic Nuclei", eds. Giuricin et al., Reidel, Dordrecht

Heckman, T.M. : 1980, Astronomy and Astrophysics, 87, 152

Stasinska, G. : 1980, Astronomy and Astrophysics, 84, 320

Stasinska, G. : 1984, Astronomy and Astrophysics, 135, 341 